\title{
GC-MS Analysis of Bioactive Compounds from Whole Plant Chloroform Extract of Ageratum conyzoides
}

\author{
Ahamefula Anse Im Ahuchaogu ${ }^{1 *}$, Godwin .I. Ogbuehi ${ }^{1}$, A I. Obike ${ }^{1}$, \\ Chisom Samps on Egedeuzu' ${ }^{1}$, Okoronkwo Joseph Chukwu ${ }^{2}$, John Bull Onyekachi Echeme ${ }^{2}$ \\ ${ }^{1}$ Department of pure and Industrial Chemistry, Abia State University, Uturu, Nigeria. \\ ${ }^{2}$ Department of Chemistry, Michael Okpara University of Agriculture, Umudike, Umuahia, Abia State, Nigeria
}

*Corresponding Author: Ahamefula Anselm Ahuchaogu, Department of pure and Industrial Chemistry, Abia State University, Uturu, Nigeria

\begin{abstract}
Most of the drug industries depend on medicinal plants for the production of pharmaceutical compounds, most herbal medicines and their derivatives products were often prepared from crude extracts from plants; which comprise a complex mixture of different phytochemical constituents (plant secondary metabolites). The aim of the work is to identify the bioactive compounds present in the chloroform extract of Ageratum conyzoides whole plant using Gas Chromatography and Mass spectroscopy. The GC-MS revealed seventy one phytochemicals ranging from high and low molecular weight chemical entities with varying quantities present. The major compounds present include 9,12-Octadecadienoic acid (12.48\%), cis-4-Acetoxytrans-1-(m-methoxyphenyl)cyclohexanecarbonitrile (11.52\%), Octadecanoic acid (4.97\%), Tetratetracontane (1.35\%), 1H-Indole-3-carboxaldehyde (3.68\%), 5-methoxy-19-Hydroxy-3-alpha,5-cyclo-5alpha-androstan17-one (1.44\%), cis-4,4-Dimethylbicyclo(6.3.0) undecane-2,6-dione (1.89\%), 2-Heptenoic acid (1.23\%), Kaur-16-ene (1.04\%), Bicyclo [11.3.0] hexadecane-2,14-dione (0.45\%), 3,3,6,6-Tetramethyl-1,2,3,4,5,6,7,8octahydroacridine (1.61\%). The minor ones such as Benzenemethanol, 4-(1,1-dimethylethyl)- (0.28\%), Benzaldehyde, 2-hydroxy-4-methoxy-(0.22\%), 2,3,4,7-Tetrahydro-1H-indene (0.18\%), 7-Oxabicyclo [4.1.0] heptanes (0.16\%), 2-Undecanone, 6,10-dimethyl-(0.96\%), Diazoacetic acid, 2 -isopropyl-5-methylcyclohexyl ester (0.54\%), Bicyclo[11.3.0] hexadecane-2,14-dione (0.45\%), are also present. These chemical compounds are considered biologically and pharmacologically important.
\end{abstract}

Keywords: Ageratum conyzoides, herbal medicine, Osu angweri ngwa, GC-MS analysis, bioactive compounds

\section{INTRODUCTION}

Medicinal plants are sources of herbal and synthetic drugs, a lot of medicine if not all, have bioactive constitutes which are responsible for biological and pharmacological activities they exhibit.

Most of the activities can be attributed to secondary metabolites (phytochemical constituents) that are bioactive and present in the plant. Secondary metabolites are organic compounds that are not directly involved in the normal growth development and reproduction of the plant [1] and through to result from the evolutionary defense put up by plants [2]. Examples include alkaloids, tannins, terpenoids, flavonoids, saponins, phenolics, coumarins, glycosides and steroids.

They are of paramount importance in the treatment of diseases for man. South Eastern Nigeria is endowed with many of such vegetative plants and they are used as food and in herbal medicine to cure diseases and heal injuries; this implies that treatment of diseases in this region is not limited to synthetic drugs, as most people still depend on botanical preparations as medicine. These are always available in form of infusions, decoctions, macerations and concoctions. Most of these people even believe that herbal preparations are more effective than conventional synthetic drugs but are more effective than conventional synthetic drugs but are skeptical about is use since there is no clear cut dosage hence making it a major constraint

Ageratum conyzoides is among the medicinal plants that have effect against disease and may contain those biologically active compounds, which are effective against ailments. It belongs to the family and tribe of Asteraceae and Eupatoriae respectively. The plant is native to Central America, Caribbean, Florida (USA), South- East Asia, South China, India, West Africa (including Vigeria), Australia and South America [3,4]. The plan is traditionally called "Ufu Opioko" and "Otogo" by the Igedes in Benue 
State, Nigeria [5]; in South Western Nigeria, it is known as "Imi esu" [6]; in South Western Nigeria, it is known as "Imi esu" [7]; while in the South East part of Nigeria, billygoat weed is called "Akwukwo nwa osi n'aka" or "Osu angweri ngwa". Ageratum conyzoides has been known since ancient times for its curative properties and has been utilized for the treatment of various ailments, such as typhoid, anaemia, malaria, headache, burns and wounds, analgesic, inflammation, asthma, spasmodic arthosis, dysnea, pneumonia and haemostatic effects, stomach ailments, gynecological diseases, leprosy and other skin diseases. [8,9]. A large percentage of the publications on the photochemistry has to do with the essential oil of this plant.

The oil content varies randomly from 0.11 to $0.58 \%$ for leaves and from 0.03 to $0.18 \%$ for the roots depending on times of the year [10]. From water distillation of the fresh flowers, the oil content was found to be $0.2 \%$. The yield of oil from the petroleum ether extract of the seed was $26 \%$ [4].

A large number of constituents have been identified from the GC-MS analysis of the essential oil of A. conyzoides. The largest so Far, a total of 51 constituents have been reported from the analysis of an oil sample of the plant collected from a university environment in Nigeria. [11]. Group of compounds like monoterpenes, sesquiterpenes, triterpenes, sterols, chromene, chromone, benzofuran, coumarins, flavonoids, alkalodis, tannins, saponins, precocene I and II and other miscellaneous compounds have been identified in Ageratum conyzoides.

\section{Materials AND Methods}

\subsection{Sample Collection and Preparation}

Fresh plant materials were collected from a nearby farmland within Michael Okpara University of Agriculture, Umudike in the month of March, 2018 and was taxonomically identified by Mr. I. Ndukwe in plant taxonomy section of the Forestry Department of Michael Okpara University of Agriculture Umudike, Nigeria.A voucher specimen (AC 7344) was deposited at Department of Botany School of biological sciences.

The Fresh leaves, stems and roots were harvested washed with tap water and rinsed with sterile distilled water, then dried under shade to prevent interference of UV-radiation from the sun. Dried plant materials were powdered using electric blender. The powdered materials were preserved in an air-tight container, ready for extraction.

\subsection{Extraction of Plant Materials}

The powdered plant sample $(500 \mathrm{~g})$ was extracted with $2 \mathrm{~L}$ of chloroform $\left(8 \mathrm{hrs} / 3\right.$ times $\left./ 30{ }^{\circ} \mathrm{c}\right)$. The extract was concentrated under reduced pressure using Digital Heidolph Rotary evaporator (4000 series) and the supernatant plant extract $(6.54 \mathrm{~g})$ was decanted after complete removal of the solvent. The extract was centrifuged at 10,000 ppm for 20 minutes and tract was then subjected to systematic GC analysis.

\subsection{GC - MS Analysis Conditions}

The GC-MS analysis of the extract was carried out using a HP 7890 GC instrument integrated with an Agilent 5975C MSD mass spectrometer (Aligent, Santa Clara, CA, USA). The capillary column was an Agilent HP-5MS (30.m x 0.25mm i.d. x 0.25 NM film thickness), helium (Purity > 99.999\%) was used as the carrier gas, and the flow rate was $1 \mathrm{ML} / \mathrm{min}$. The injector temperature was $250^{\circ} \mathrm{c}$, and the injection mode was splitless. The G.C oven temperature was held at $50^{\circ} \mathrm{C}$ for $5 \mathrm{~min}$, which was increased to $210^{\circ} \mathrm{c}$ at a rate of $3^{\circ} \mathrm{C} / \mathrm{min}$, maintained at $210^{\circ} \mathrm{c}$ for $3 \mathrm{~min}$, and finally increased to $230^{\circ} \mathrm{C}$ at $150 \mathrm{C} / \mathrm{min}$. The mass spectrometer conditions were as follow: $[12,13,14]$ ionization energy, $70 \mathrm{Ev}$; ion Source temperature, $230^{\circ} \mathrm{C}$; quadrupole temperature, $150^{\circ} \mathrm{C}$; quadrupole mass spectrometer scan range $30-$ 500 atomic mass units (amu); solvent delay time $2.8 \mathrm{~min}$.

\subsection{Components Identification}

The components of the chloroform extract of Ageratum conyzoides was identified by matching the peaks with computer Wiley Ms. libraries and confirmed by comparing mass spectra of the peaks and those from literature [15].

\section{RES ULTS AND DIS CUS S IONS}

The chloroform extract of the whole plant of Ageratum conyzoides on GC-MS analysis showed seventy one peaks indicating the presence of seventy one compounds in the plant as shown in figure 1. 
The molecular formula, the molecular weight, the retention time and the percentage constituents of the compounds are shown in Table 1.

The mass spectrometer analyzes the compounds eluted at different times help to identify the nature and structures of the compounds. The large compounds fragments into small compounds giving rise to appearance of peaks at different $\mathrm{m} / \mathrm{z}$ ratios. These mass spectra are fingerprint of that compounds which can be identified from the data library. The GC-MS study of the chloroform extract of the whole plant of Ageratum conyzoide had shown the presence of lots of photochemical which strongly contribute to the medicinal activity of the whole plant. The identified major compounds possess some important biological potential for future drug development

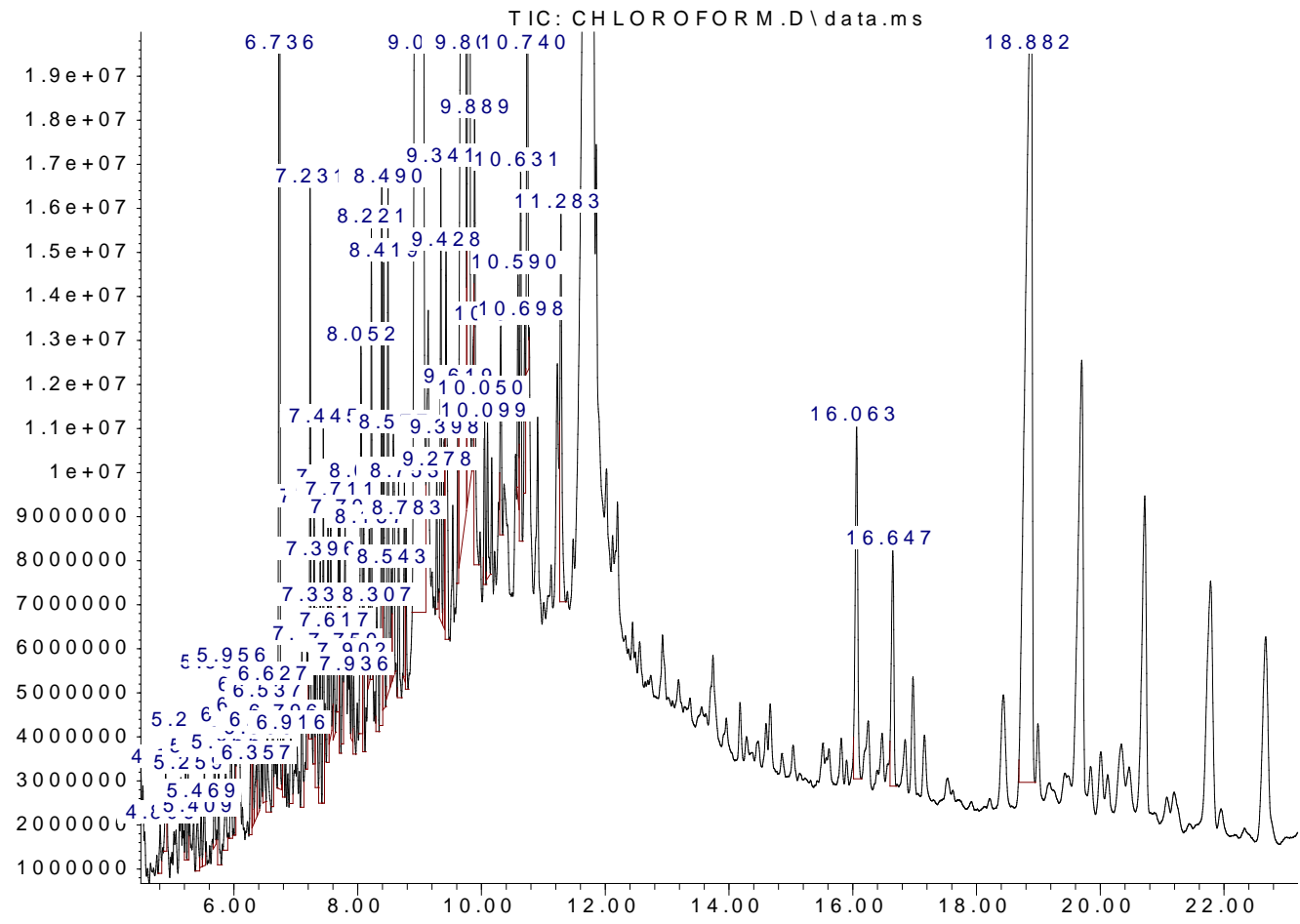

Tim e-->

Figure1. GC-MS Chromatogram of Ageratum conyzoides whole plant chloroform extract.

Table1. GC - MS analysis of Ageratum conyzoides showing molecular formula, molecular weight, percentage content, retention time

\begin{tabular}{|c|c|l|l|c|c|}
\hline SN & RT & COMPONENT & FORMULA & MW & \% \\
\hline 1 & 4.806 & 7-Oxabicyclo[4.1.0]heptanes & $\mathrm{C}_{6} \mathrm{H}_{10} \mathrm{O}$ & 98 & 0.16 \\
\hline 2 & 4.895 & Octanoic Acid & $\mathrm{C}_{8} \mathrm{H}_{16} \mathrm{O}_{2}$ & 144 & 0.26 \\
\hline 3 & 5.218 & Azulene & $\mathrm{C}_{10} \mathrm{H}_{8}$ & 128 & 0.25 \\
\hline 4 & 5.259 & 2,3,4,7-Tetrahydro-1H-indene & $\mathrm{C}_{9} \mathrm{H}_{12}$ & 120 & 0.18 \\
\hline 5 & 5.409 & Benzeneacetic acid & $\mathrm{C}_{8} \mathrm{H}_{8} \mathrm{O}_{2}$ & 136 & 0.23 \\
\hline 6 & 5.469 & n-Decanoic acid & $\mathrm{C}_{10} \mathrm{H}_{20} \mathrm{O}_{2}$ & 172 & 0.16 \\
\hline 7 & 5.521 & 2-Decenal, (Z)- & $\mathrm{C}_{10} \mathrm{H}_{18} \mathrm{O}$ & 154 & 0.28 \\
\hline 8 & 5.686 & Phenol, 2-methyl-5-(1-methylethyl) & $\mathrm{C}_{10} \mathrm{H}_{14} \mathrm{O}$ & 150 & 0.36 \\
\hline 9 & 5.754 & Phenol, 2,3,5,6-tetramethyl- & $\mathrm{C}_{10} \mathrm{H}_{14} \mathrm{O}$ & 150 & 0.27 \\
\hline 10 & 5.866 & 2-Methoxy-4-vinylphenol & $\mathrm{C}_{9} \mathrm{H}_{10} \mathrm{O}_{2}$ & 150 & 0.32 \\
\hline 11 & 5.956 & 2-Acetylcyclopentanone & $\mathrm{C}_{7} \mathrm{H}_{10} \mathrm{O}_{2}$ & 126 & 0.44 \\
\hline 12 & 6.020 & Benzaldehyde, 2-hydroxy-4-methoxy- & $\mathrm{C}_{8} \mathrm{H}_{8} \mathrm{O}_{3}$ & 152 & 0.22 \\
\hline 13 & 6.279 & 1,3-Propanediol, 2,2-diethyl- & $\mathrm{C}_{7} \mathrm{H}_{16} \mathrm{O}_{2}$ & 132 & 0.36 \\
\hline 14 & 6.301 & 2-Cyclohexen-1-ol, 2-methyl-5-(1-methylethenyl)-, cis & $\mathrm{C}_{10} \mathrm{H}_{16} \mathrm{O}$ & 152 & 0.27 \\
\hline 15 & 6.357 & 2-Butenoic acid, 3-methyl- & $\mathrm{C}_{5} \mathrm{H}_{8} \mathrm{O}_{2}$ & 100 & 0.19 \\
\hline 16 & 6.395 & Cyclohexanol, 1-(aminomethyl)- & $\mathrm{C}_{7} \mathrm{H}_{15} \mathrm{NO}_{2}$ & 129 & 0.19 \\
\hline 17 & 6.481 & 3-Furanmethanol & $\mathrm{C}_{5} \mathrm{H}_{6} \mathrm{O}_{2}$ & 98 & 0.21 \\
\hline 18 & 6.537 & 1H-Pyrazole, 3-ethoxy-5-methyl- & $\mathrm{C}_{6} \mathrm{H}_{10} \mathrm{~N}_{2} \mathrm{O}$ & 126 & 0.33 \\
\hline
\end{tabular}




\begin{tabular}{|c|c|c|c|c|c|}
\hline 19 & 6.627 & Phenol, 2-methoxy-4-(1-propenyl)-, (E) & $\mathrm{C}_{10} \mathrm{H}_{12} \mathrm{O}_{2}$ & 164 & 0.25 \\
\hline 20 & 6.736 & 1H-Indole-3-carboxaldehyde, 5-methoxy & $\mathrm{C}_{10} \mathrm{H}_{9} \mathrm{NO}_{2}$ & 175 & 3.68 \\
\hline 21 & 6.796 & $\begin{array}{l}\text { Furan, 2,5-dihydro-2,2-dimethyl-5-(1-methylethenyl)-3-(1- } \\
\text { methylethyl)- }\end{array}$ & $\mathrm{C}_{12} \mathrm{H}_{20} \mathrm{O}$ & 180 & 0.19 \\
\hline 22 & 6.916 & Hexanoic acid, 6-bromo- & $\mathrm{C}_{6} \mathrm{H}_{11} \mathrm{BrO}_{2}$ & 194 & 0.30 \\
\hline 23 & 7.100 & Dodecanoic acid & $\mathrm{C}_{12} \mathrm{H}_{24} \mathrm{O}_{2}$ & 200 & 0.42 \\
\hline 24 & 7.178 & $\alpha$-Calacorene & $\mathrm{C}_{15} \mathrm{H}_{20}$ & 200 & 0.28 \\
\hline 25 & 7.231 & 2H-1-Benzopyran-2-one, 6-hydroxy-7-methoxy-4-methyl- & $\mathrm{C}_{11} \mathrm{H}_{10} \mathrm{O}_{4}$ & 206 & 1.07 \\
\hline 26 & 7.295 & Tetradecane & $\mathrm{C}_{14} \mathrm{H}_{30}$ & 198 & 0.48 \\
\hline 27 & 7.332 & N,N-Diethyl-p-nitroaniline & $\mathrm{C}_{10} \mathrm{H}_{14} \mathrm{~N}_{2} \mathrm{O}_{2}$ & 194 & 0.56 \\
\hline 28 & 7.396 & (-)-Spathulenol & $\mathrm{C}_{15} \mathrm{H}_{24} \mathrm{O}$ & 220 & 0.55 \\
\hline 29 & 7.445 & Caryophyllene oxide & $\mathrm{C}_{15} \mathrm{H}_{24} \mathrm{O}$ & 220 & 0.79 \\
\hline 30 & 7.520 & 3-Buten-2-ol, 2-methyl- & $\mathrm{C}_{5} \mathrm{H}_{10} \mathrm{O}$ & 86 & 0.59 \\
\hline 31 & 7.564 & Cyclohexanol, 5-methyl-2-(1-methylethyl)-, $(1 \alpha, 2 \alpha, 5 \beta)-$ & $\mathrm{C}_{10} \mathrm{H}_{20} \mathrm{O}$ & 156 & 0.59 \\
\hline 32 & 7.617 & Methyl 7-(2-furyl)heptanoate & $\mathrm{C}_{12} \mathrm{H}_{18} \mathrm{O}_{3}$ & 210 & 0.24 \\
\hline 33 & 7.688 & 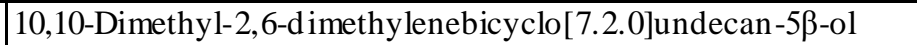 & $\mathrm{C}_{15} \mathrm{H}_{24} \mathrm{O}$ & 220 & 0.50 \\
\hline 34 & 7.711 & Pyrimidine, 4-(2-hydroxy-5-metho xyphenyl)- & $\mathrm{C}_{11} \mathrm{H}_{10} \mathrm{~N}_{2} \mathrm{O}_{2}$ & 202 & 0.43 \\
\hline 35 & 7.759 & Methyl diphenylphosphinite & $\mathrm{C}_{13} \mathrm{H}_{13} \mathrm{OP}$ & 216 & 0.24 \\
\hline 36 & 7.797 & Limonene oxide, cis- & $\mathrm{C}_{10} \mathrm{H}_{16} \mathrm{O}$ & 152 & 0.32 \\
\hline 37 & 7.902 & $\begin{array}{l}\text { 2-Methyl-3-(3-methyl-but-2-enyl)-2-(4-methyl-pent-3-enyl)- } \\
\text { oxetane }\end{array}$ & $\mathrm{C}_{15} \mathrm{H}_{26} \mathrm{O}$ & 222 & 0.16 \\
\hline 38 & 7.936 & Longipinocarveol, trans- & $\mathrm{C}_{15} \mathrm{H}_{24} \mathrm{O}$ & 220 & 0.16 \\
\hline 39 & 8.052 & Tetradecanoic acid & $\mathrm{C}_{14} \mathrm{H}_{28} \mathrm{O}_{2}$ & 228 & 1.35 \\
\hline 40 & 8.097 & 6-Amino-2,4-dimethyl-5-metho xyquinoline & $\mathrm{C}_{12} \mathrm{H}_{14} \mathrm{~N}_{2} \mathrm{O}$ & 202 & 0.51 \\
\hline 41 & 8.187 & Carbonic acid, octadecyl 2,2,2-trichloroethyl ester & $\mathrm{C}_{21} \mathrm{H}_{39} \mathrm{Cl}_{3} \mathrm{O}_{3}$ & 444 & 0.36 \\
\hline 42 & 8.220 & Heptadecane & $\mathrm{C}_{17} \mathrm{H}_{36}$ & 240 & 0.80 \\
\hline 43 & 8.307 & Norfenefrine & $\mathrm{C}_{8} \mathrm{H}_{11} \mathrm{NO}_{2}$ & 153 & 0.44 \\
\hline 44 & 8.389 & 1,19-Eicosadiene & $\mathrm{C}_{20} \mathrm{H}_{38}$ & 278 & 1.36 \\
\hline 45 & 8.419 & 2-Undecanone, 6,10-dimethyl- & $\mathrm{C}_{13} \mathrm{H}_{26} \mathrm{O}$ & 198 & 0.96 \\
\hline 46 & 8.490 & Pentadecanoic acid & $\mathrm{C}_{15} \mathrm{H}_{30} \mathrm{O}_{2}$ & 242 & 1.31 \\
\hline 47 & 8.543 & Benzenemethanol, 4-(1,1-dimethylethyl)- & $\mathrm{C}_{11} \mathrm{H}_{16} \mathrm{O}$ & 164 & 0.28 \\
\hline 48 & 8.577 & 3,7,11,15-Tetra methyl-2-he xadecen-1-ol & $\mathrm{C}_{20} \mathrm{H}_{40} \mathrm{O}$ & 296 & 0.81 \\
\hline 49 & 8.663 & 4-Allyl-5-pyridin-3-y 1-2,4-dihydro-[1,2,4]triazo le-3-thione & $\mathrm{C}_{10} \mathrm{H}_{10} \mathrm{~N}_{4} \mathrm{~S}$ & 218 & 0.54 \\
\hline 50 & 8.753 & Pentadecanoic acid, 14-methyl-, methyl ester & $\mathrm{C}_{17} \mathrm{H}_{34} \mathrm{O}_{2}$ & 270 & 0.43 \\
\hline 51 & 8.783 & 7,9-Di-tert-butyl-1-o xaspiro(4,5)deca-6,9-diene-2,8-dione & $\mathrm{C}_{17} \mathrm{H}_{24} \mathrm{O}_{3}$ & 276 & 0.31 \\
\hline 52 & 9.041 & n-Hexadecanoic acid & $\mathrm{C}_{16} \mathrm{H}_{32} \mathrm{O}_{2}$ & 256 & 30.69 \\
\hline 53 & 9.278 & Methyl 2-hydroxy-pentadecanoate & $\mathrm{C}_{16} \mathrm{H}_{32} \mathrm{O}_{3}$ & 272 & 0.30 \\
\hline 54 & 9.341 & Heptadecanoic acid & $\mathrm{C}_{17} \mathrm{H}_{34} \mathrm{O}_{2}$ & 270 & 1.07 \\
\hline 55 & 9.398 & 1,4-Methanonaphthalen-9-ol, 1,2,3,4-tetrahydro-, stereoisomer & $\mathrm{C}_{11} \mathrm{H}_{12} \mathrm{O}$ & 160 & 0.47 \\
\hline 56 & 9.428 & 1-Hexadecene & $\mathrm{C}_{16} \mathrm{H}_{32}$ & 224 & 1.32 \\
\hline 57 & 9.619 & Kaur-16-ene & $\mathrm{C}_{20} \mathrm{H}_{32}$ & 272 & 0.44 \\
\hline 58 & 9.720 & 9,12-Octadecadienoic acid (Z,Z)- & $\mathrm{C}_{18} \mathrm{H}_{32} \mathrm{O}_{2}$ & 280 & 12.48 \\
\hline 59 & 9.806 & Octadecanoic acid & $\mathrm{C}_{18} \mathrm{H}_{36} \mathrm{O}_{2}$ & 284 & 4.97 \\
\hline 60 & 9.889 & Tetratetracontane & $\mathrm{C}_{44} \mathrm{H}_{9} \mathrm{O}$ & 618 & 1.19 \\
\hline 61 & 10.050 & (R)-(-)-14-Methyl-8-he xadecyn-1-ol & $\mathrm{C}_{17} \mathrm{H}_{32} \mathrm{O}$ & 252 & 0.43 \\
\hline 62 & 10.099 & Bicyclo[5.2.0]nonane, 4-methylene-2,8,8-trimethyl-2-vinyl- & $\mathrm{C}_{15} \mathrm{H}_{24}$ & 204 & 0.39 \\
\hline 63 & 10.312 & Bicyclo[5.2.0]nonane, 4-methylene-2,8,8-trimethyl-2-vinyl- & $\mathrm{C}_{15} \mathrm{H}_{24}$ & 204 & 0.60 \\
\hline 64 & 10.590 & Diazoacetic acid, 2-isopropyl-5-methylcyclohexyl ester & $\mathrm{C}_{12} \mathrm{H}_{20} \mathrm{~N}_{2} \mathrm{O}_{2}$ & 224 & 0.54 \\
\hline 65 & 10.631 & Kaur-16-ene & $\mathrm{C}_{20} \mathrm{H}_{32}$ & 272 & 1.04 \\
\hline 66 & 10.698 & Bicyclo[11.3.0]he xadecane-2,14-dione & $\mathrm{C}_{16} \mathrm{H}_{26} \mathrm{O}_{2}$ & 250 & 0.45 \\
\hline 67 & 10.740 & 3,3,6,6-Tetra methyl-1,2,3,4,5,6,7,8-octahydroacridine & $\mathrm{C}_{17} \mathrm{H}_{25} \mathrm{~N}$ & 243 & 1.61 \\
\hline 68 & 11.283 & 19-Hydroxy-3alpha,5-cyclo-5alpha-androstan-17-one & $\mathrm{C}_{19} \mathrm{H}_{28} \mathrm{O}_{2}$ & 288 & 1.44 \\
\hline 69 & 16.063 & cis-4,4-Dimethylbicyclo(6.3.0)undecane-2,6-dione & $\mathrm{C}_{13} \mathrm{H}_{20} \mathrm{O}_{2}$ & 208 & 1.89 \\
\hline 70 & 16.647 & 2-Heptenoic acid, 4-cyclopropyl-5-methylene-, methyl ester,(E)- & $\mathrm{C}_{12} \mathrm{H}_{18} \mathrm{O}_{2}$ & 194 & 1.23 \\
\hline 71 & 18.882 & $\begin{array}{l}\text { cis-4-Acetoxy-trans-1-(m-metho xyphenyl) } \\
\text { cyclohexanecarbonitrile }\end{array}$ & $\mathrm{C}_{16} \mathrm{H}_{19} \mathrm{NO}_{3}$ & 273 & 11.52 \\
\hline
\end{tabular}



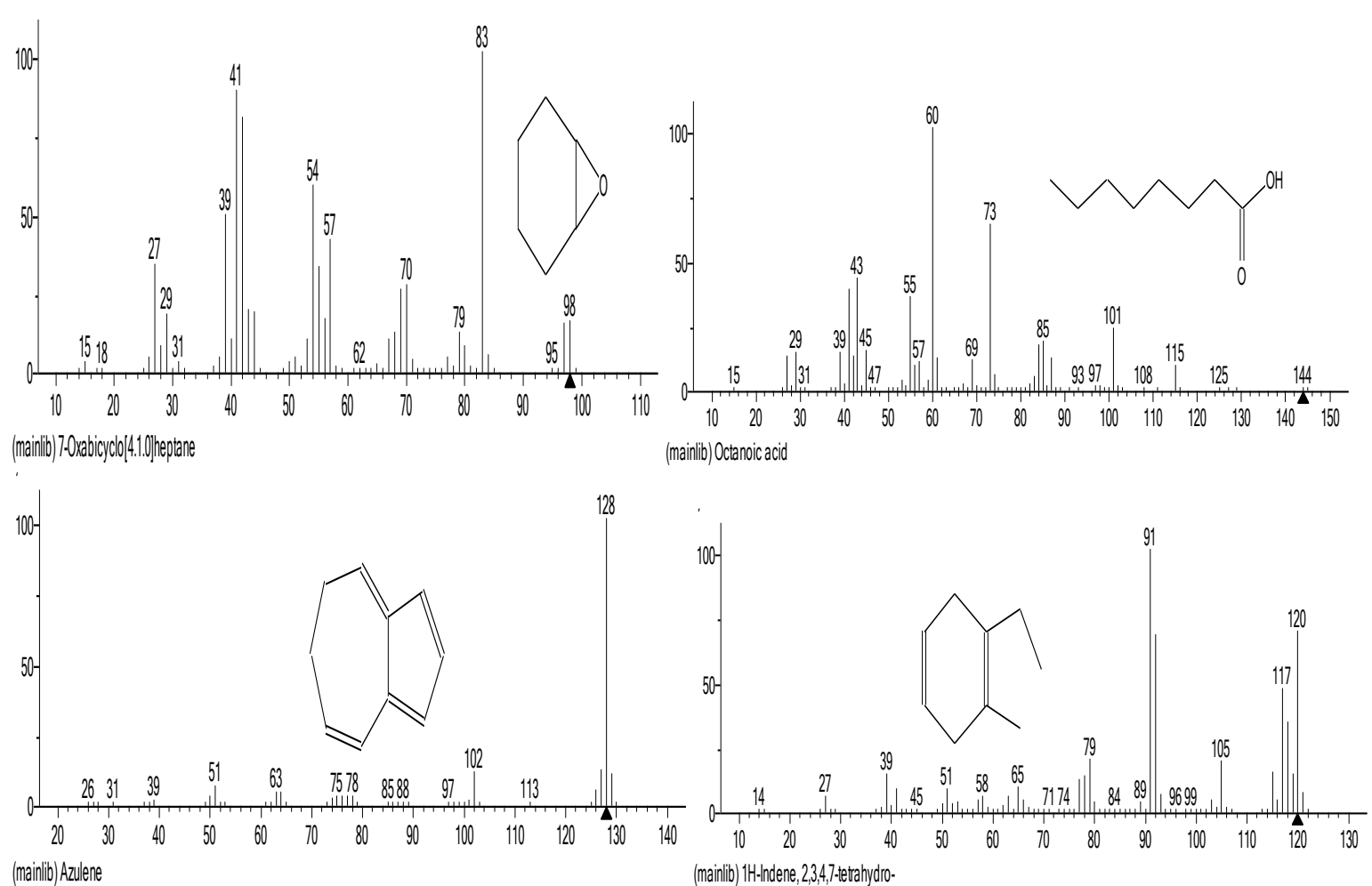
(mainilib) Azulene
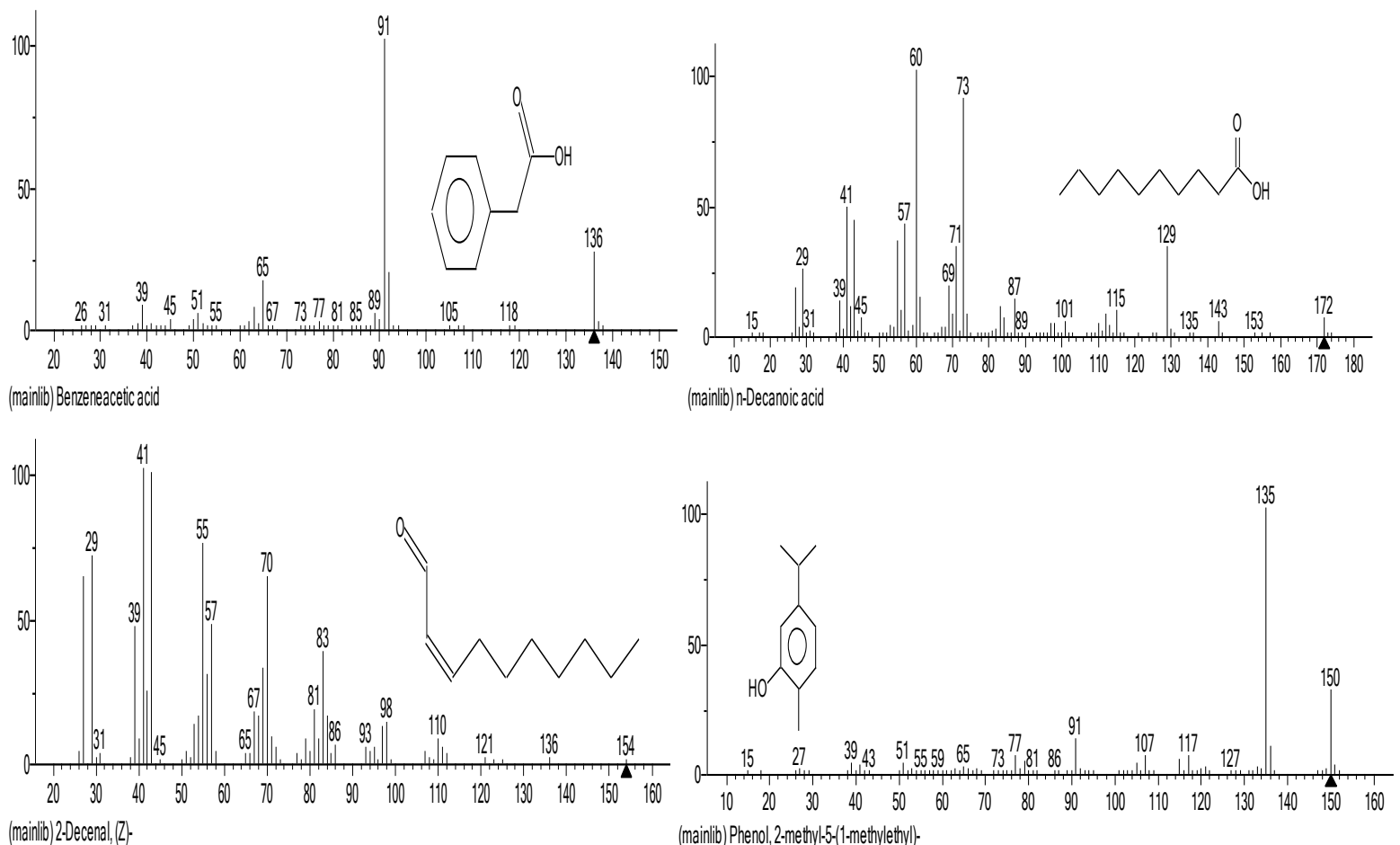
(manilib) 2-Decenal, (Z) (mainili) Pheno, 2-methyl-5-1-1-methylethy).
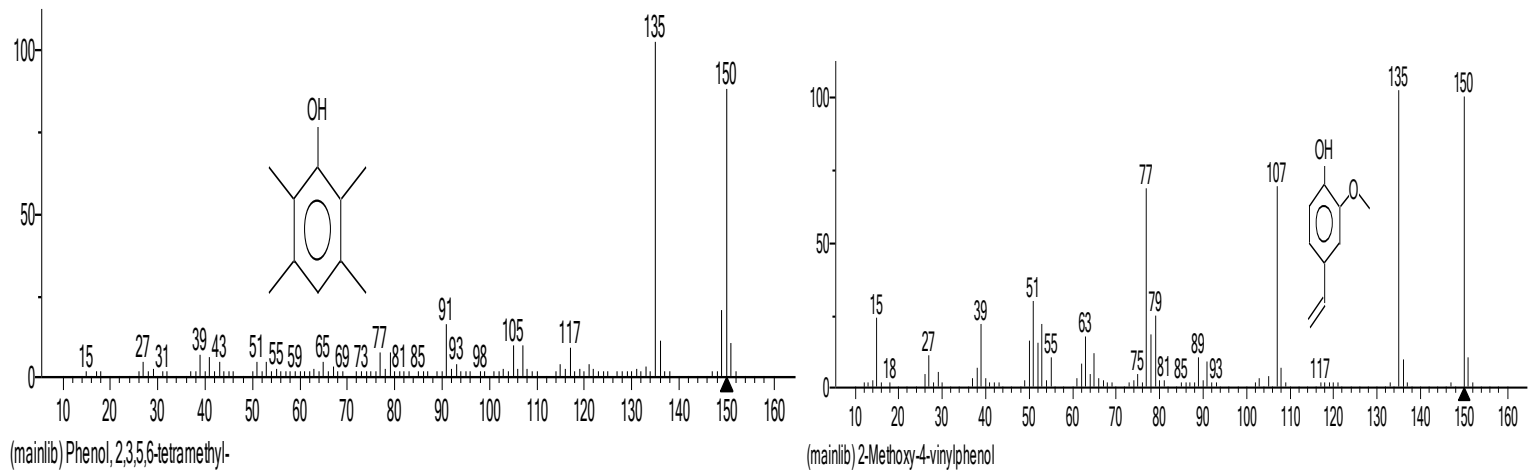
(mainilib) Pheno, 23,5,5,-tetamethyl. 

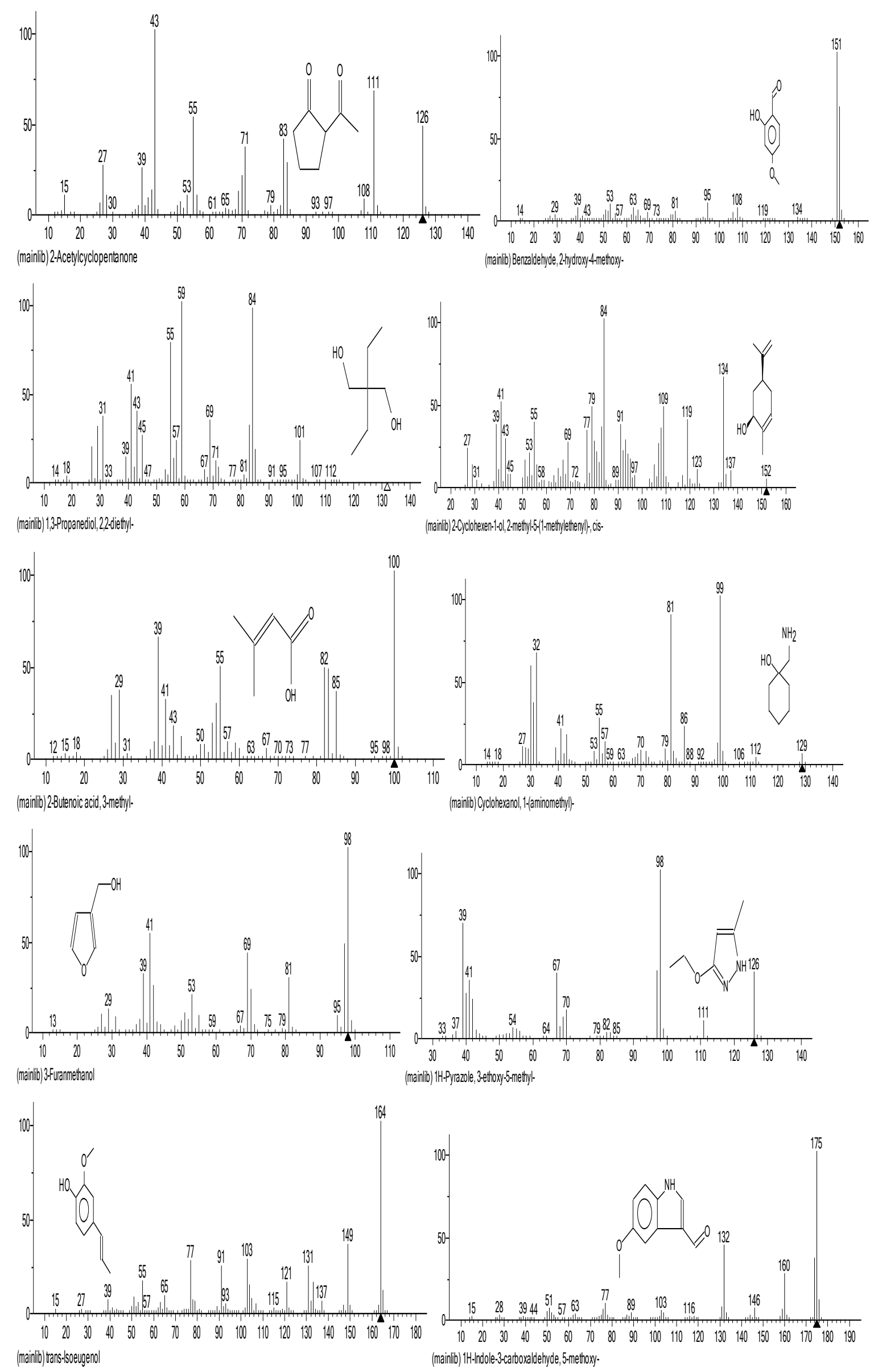

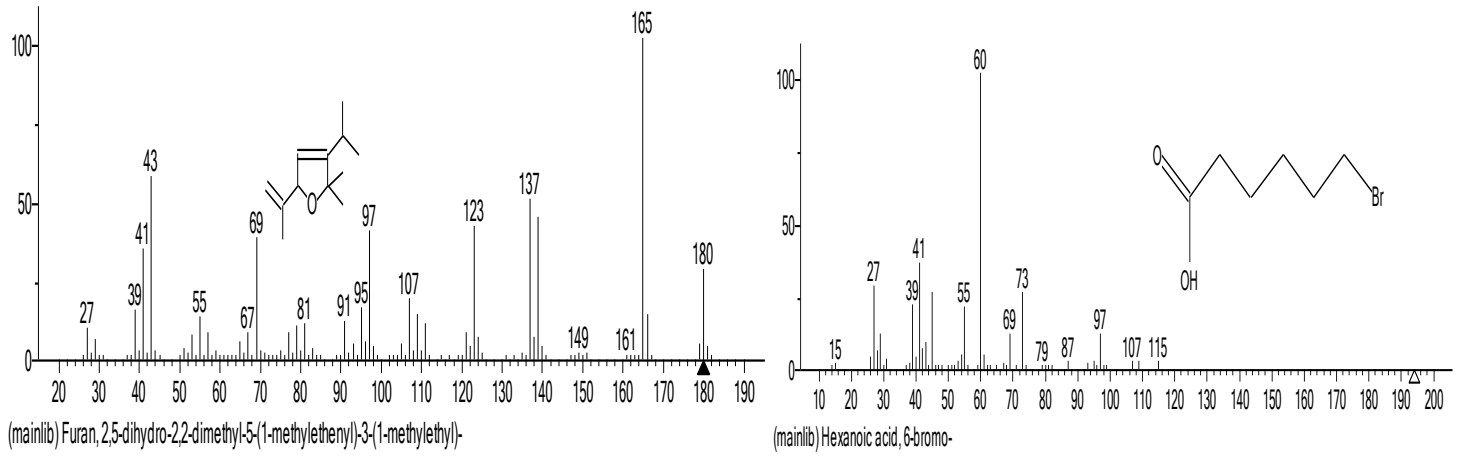

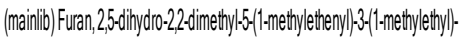
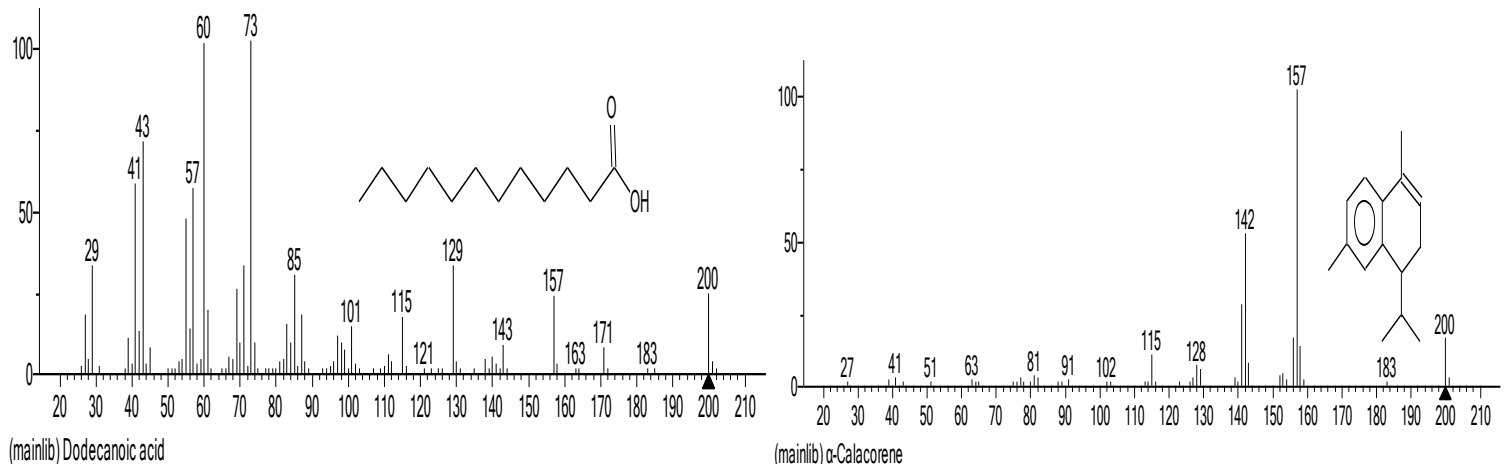

(mainili) Dodecenonic acid

$$
\text { (mainib)|a:Calacorene }
$$
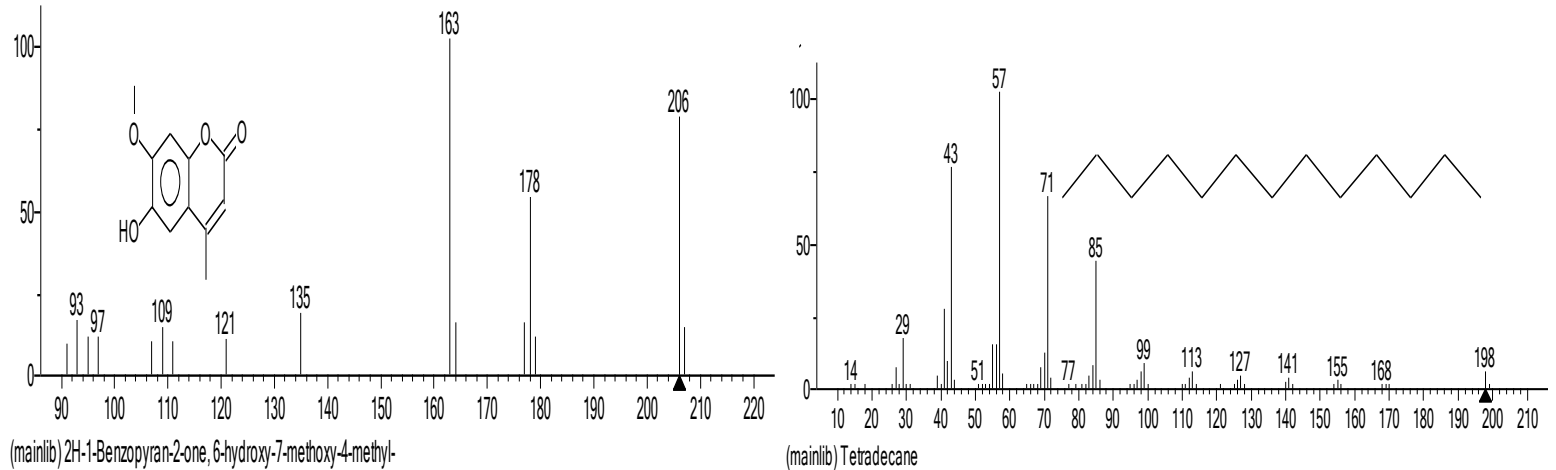

(manilib) 2H-1.Benzopyran-2-0ne, 6-hydroxy-7methoxy-4-methyl-

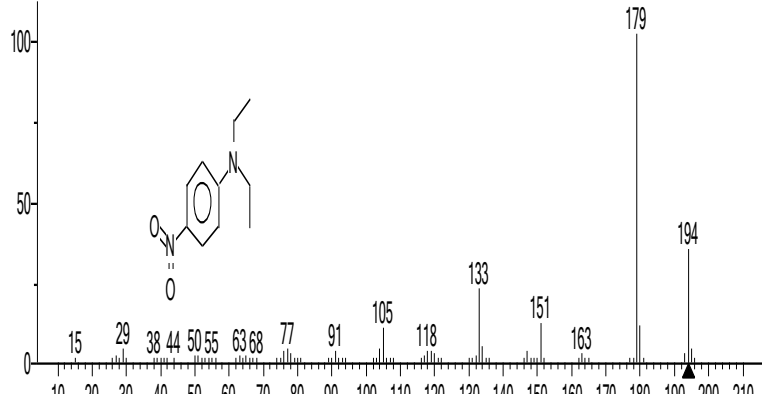

$10 \quad 20 \quad 3040 \quad 50 \quad 60 \quad 70 \quad 80 \quad 90 \quad 100110120130140150160170180190200210$ (mainibi) N,N-Diettyl.p-ritioaniline
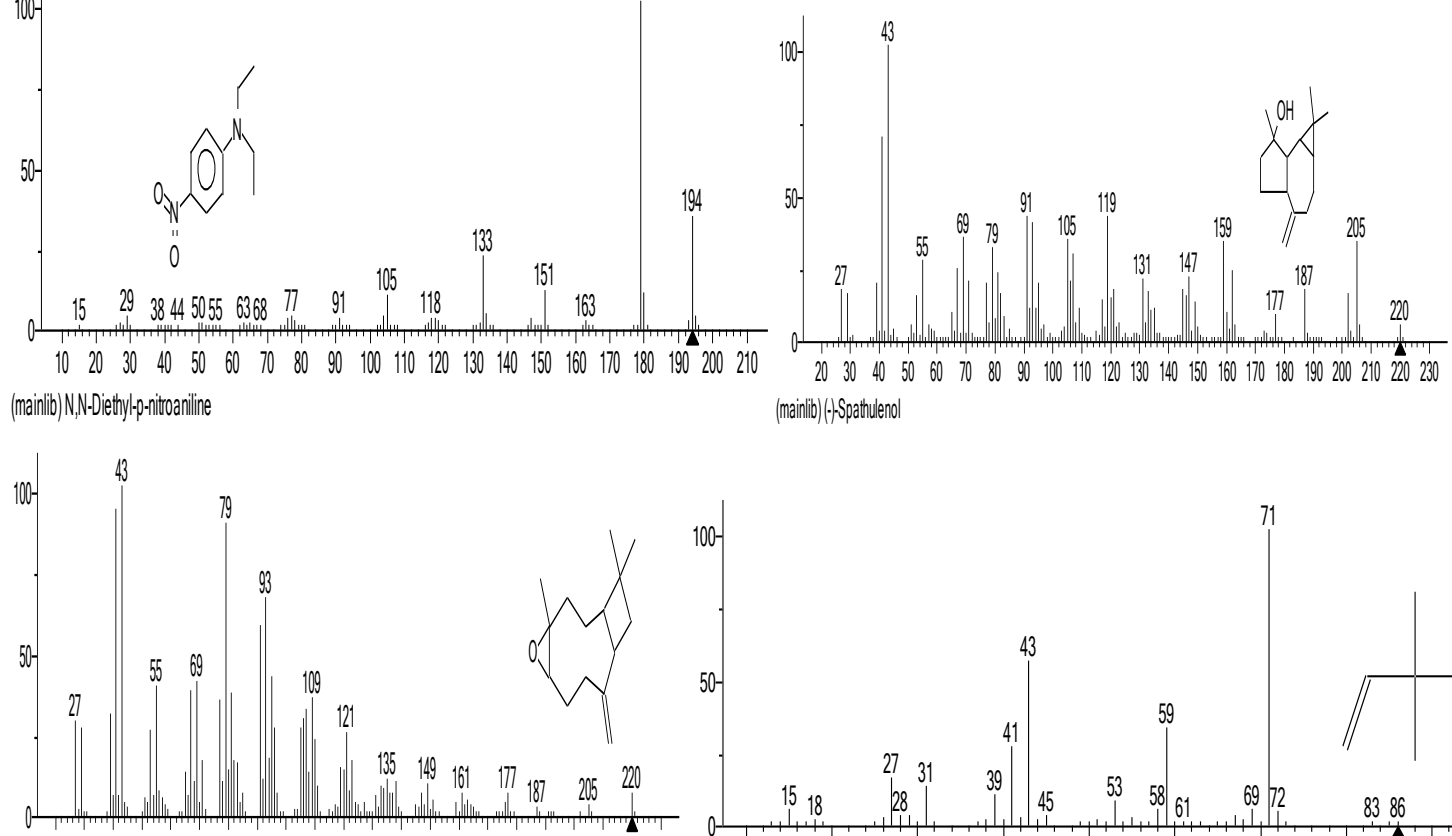

$20304050 \quad 0070 \quad 809010011012013014015010017018019020221020230$ (manilib) Carvophyllene oxide

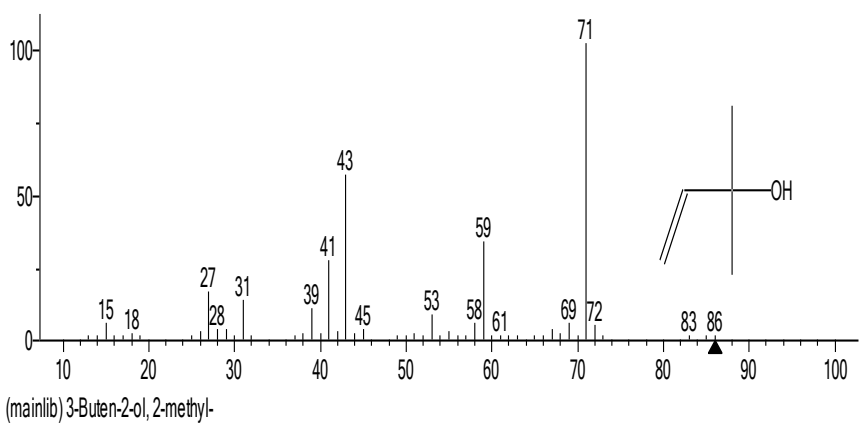



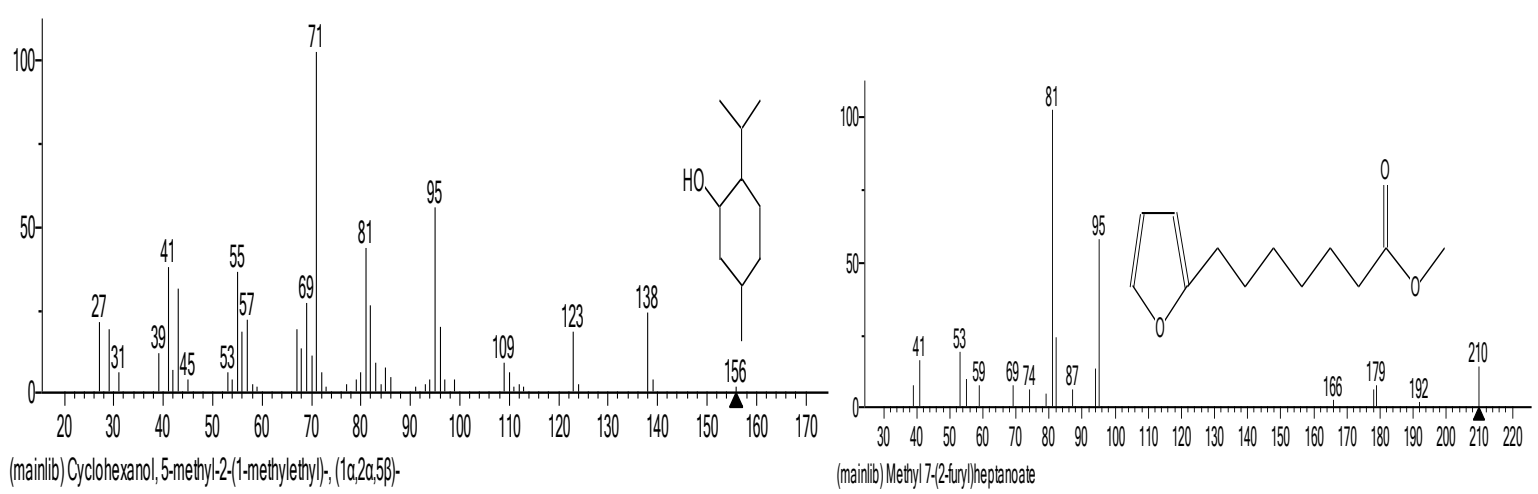

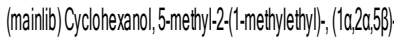

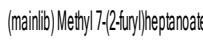

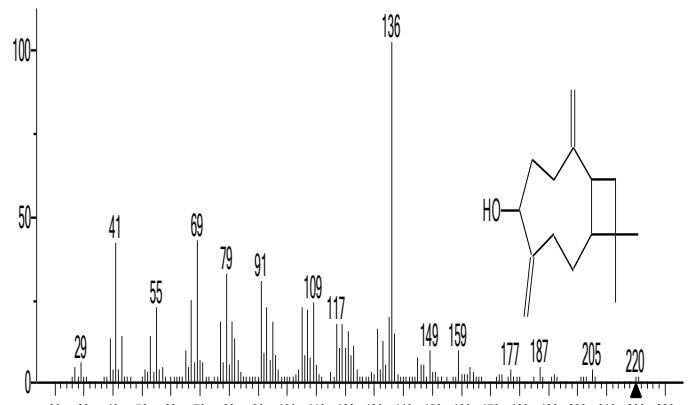

$20304050 \quad 0070 \quad 809010011012013014015010017018019020021020230$

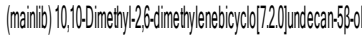
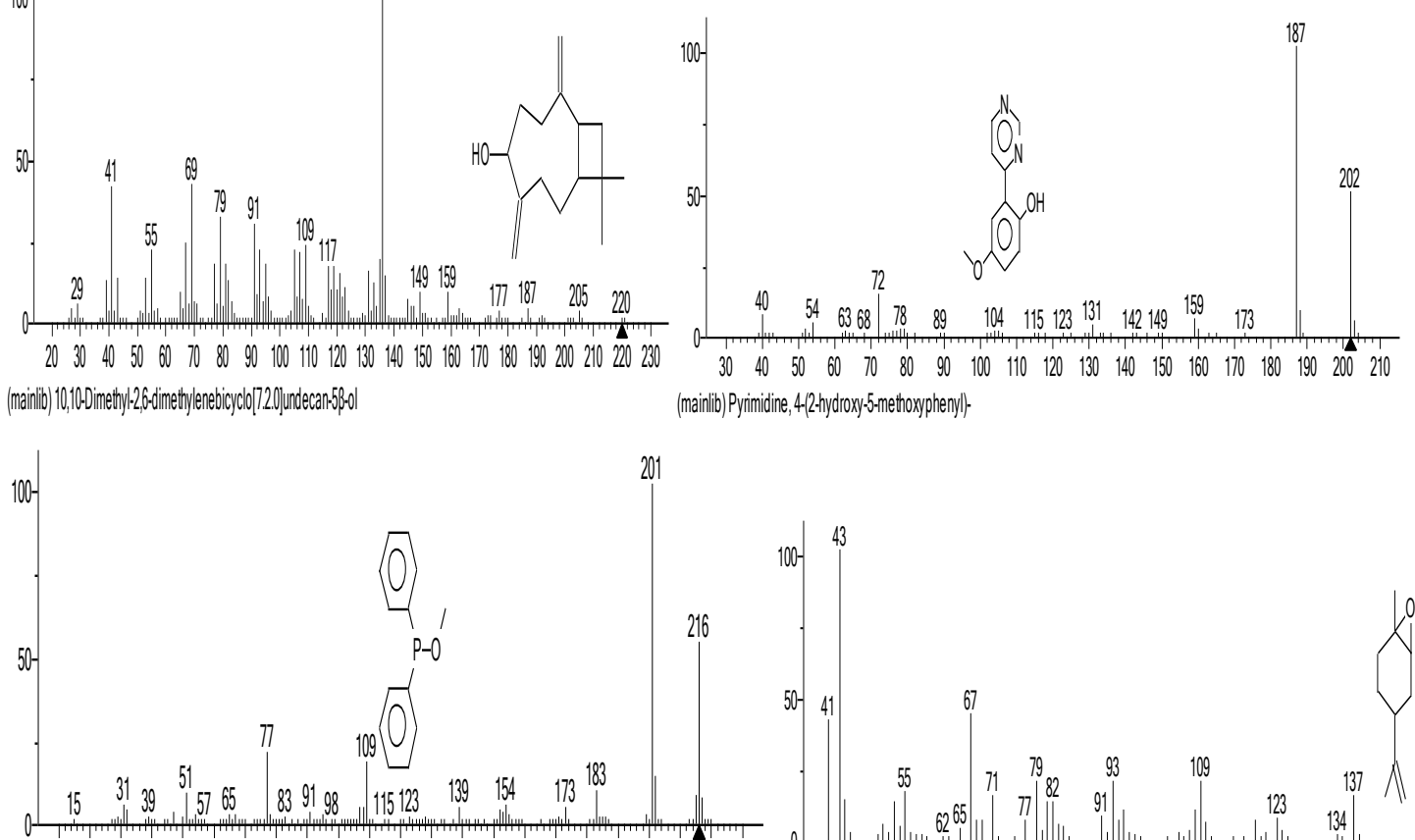

$1020304050 \quad 6070 \quad 8090100110120130140150160170180190200210220230$ (mainib) Methyl diphenylphosphinite
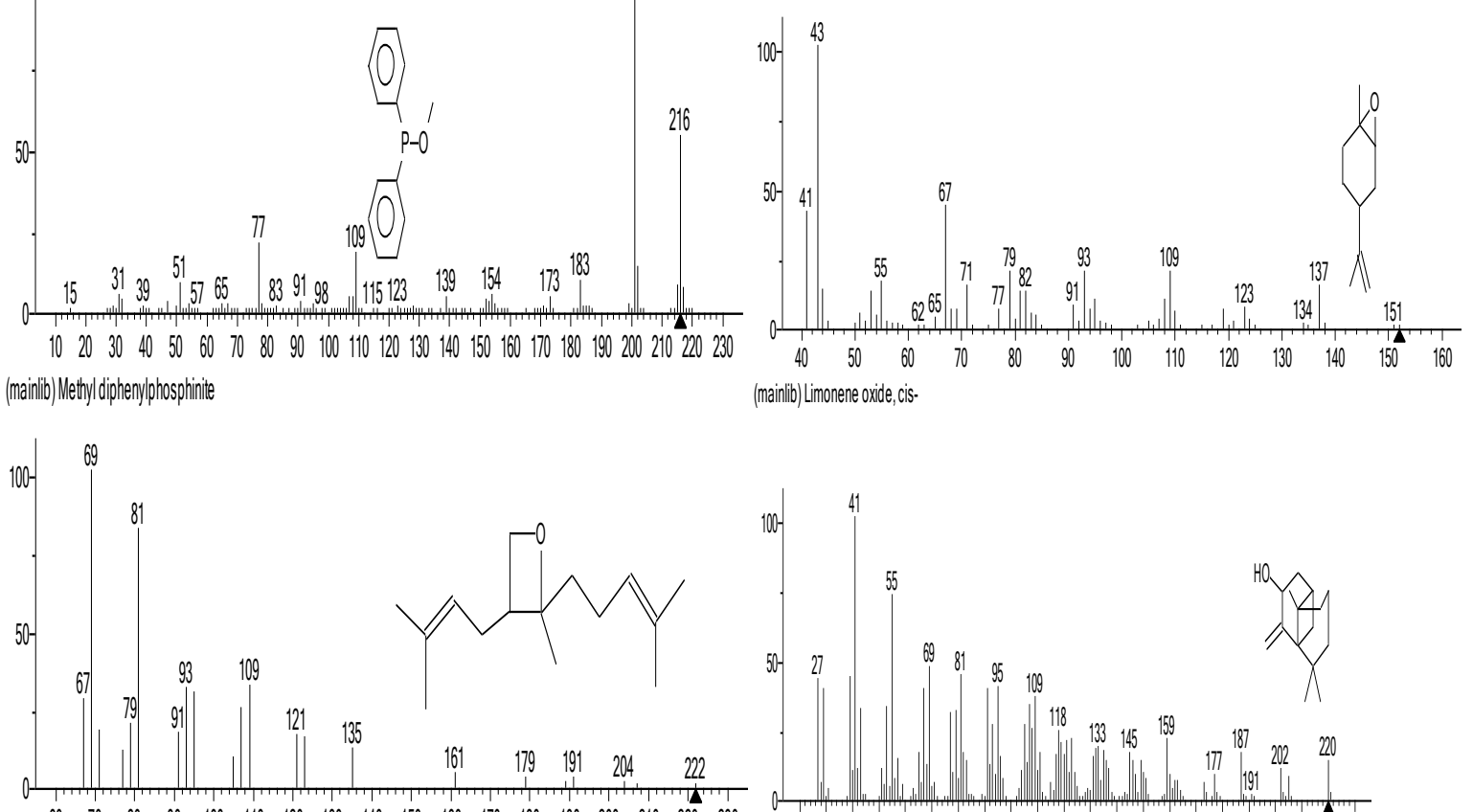

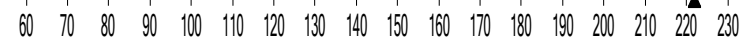

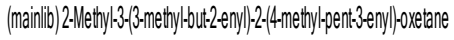
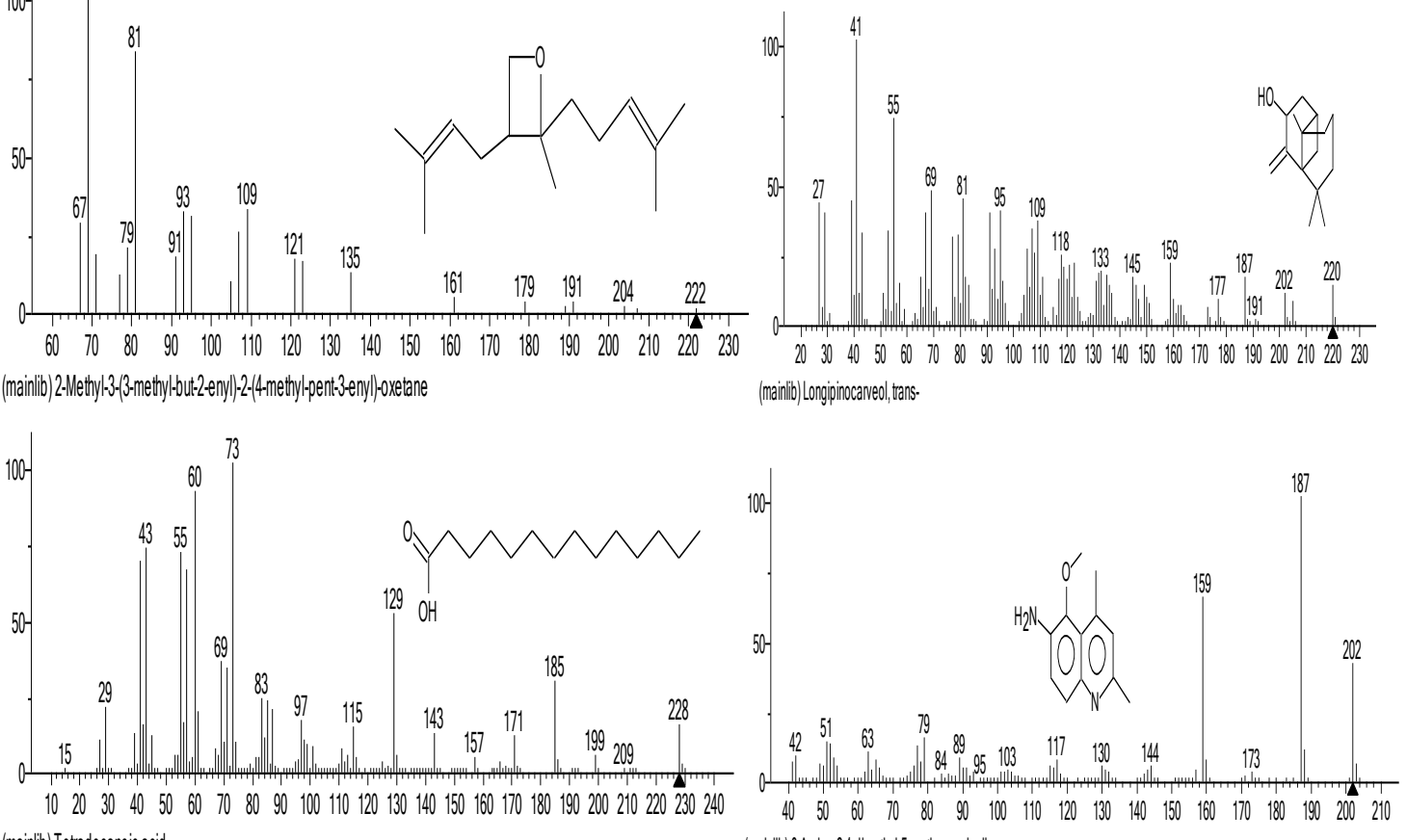
(mainili) Tetradecanoic acid

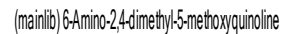



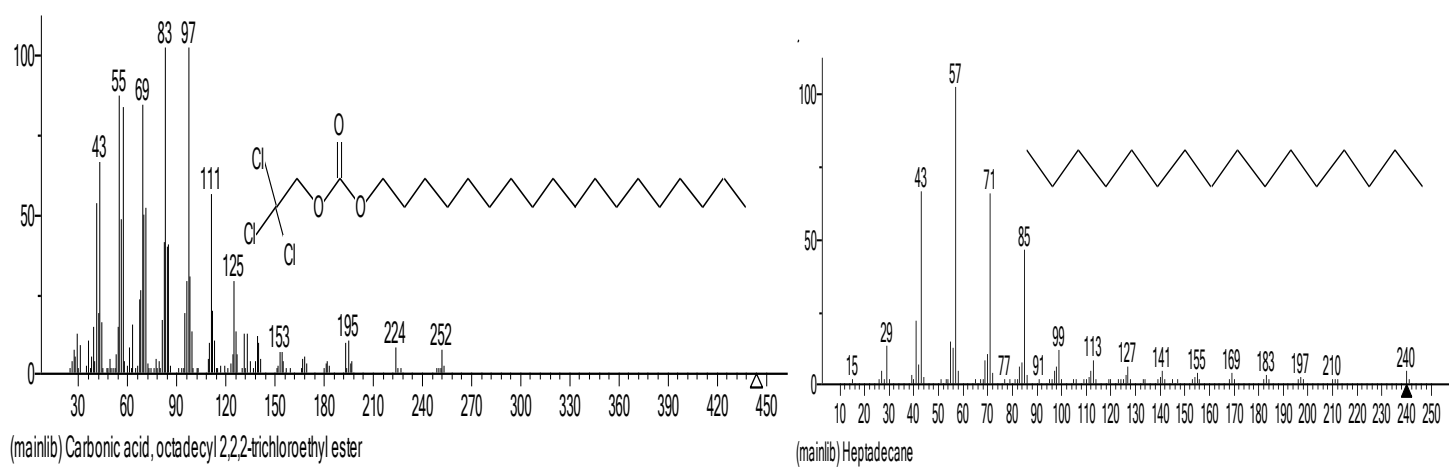

(manilib) Carbonic acid, octadecy) 2,2,-tichloroethyl ester
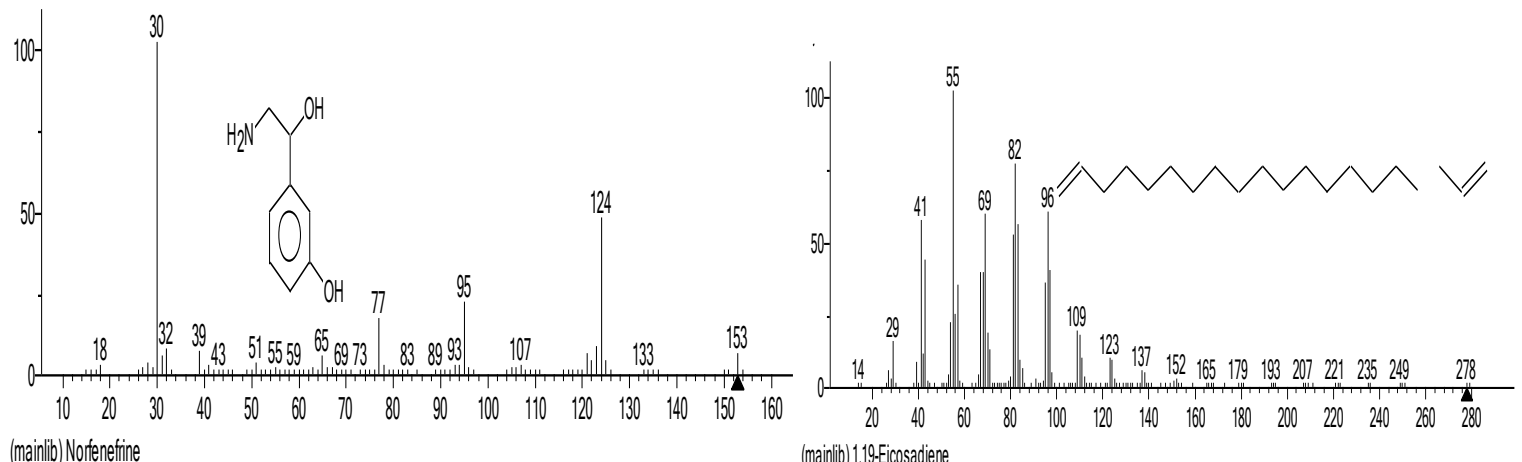
(mainilib) Norenentine

(mainili) 1,19-Eicosadiene

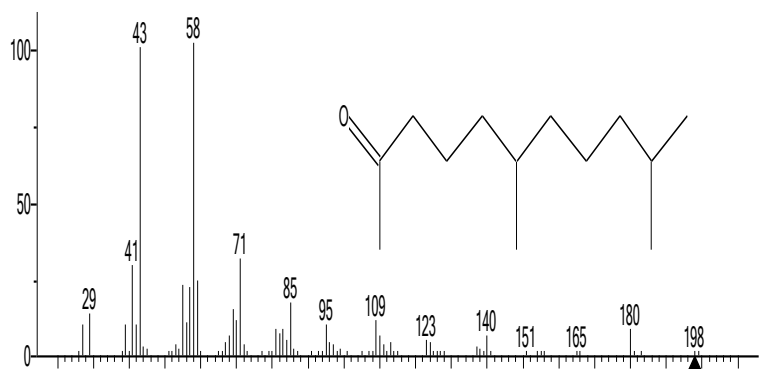

$20 \quad 3040 \quad 50 \quad 60 \quad 70 \quad 80 \quad 90 \quad 100 \quad 110 \quad 120130140150160170 \quad 180190200210$ (mainili)2:Undecanone, 6,10-dimethyl.
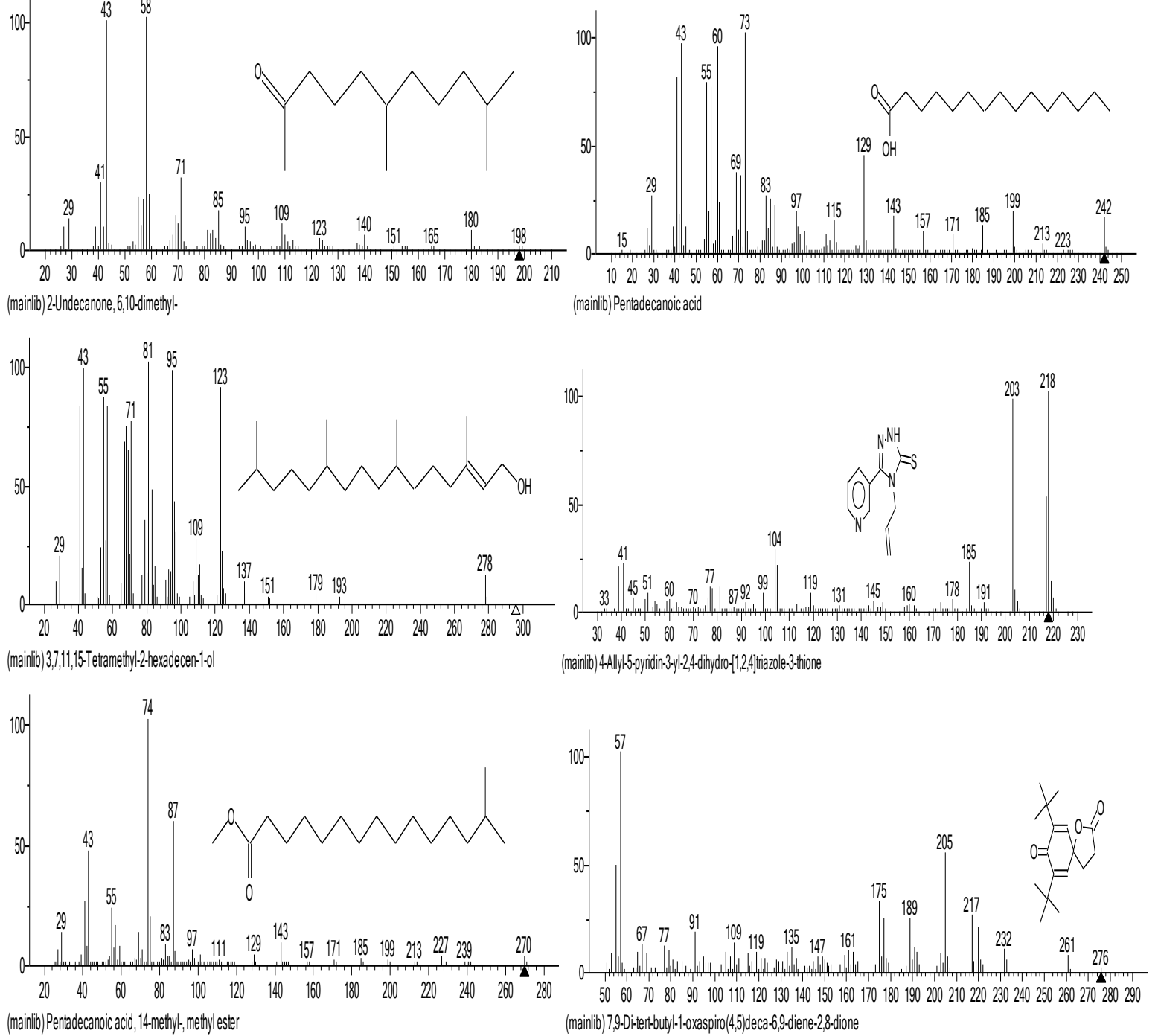

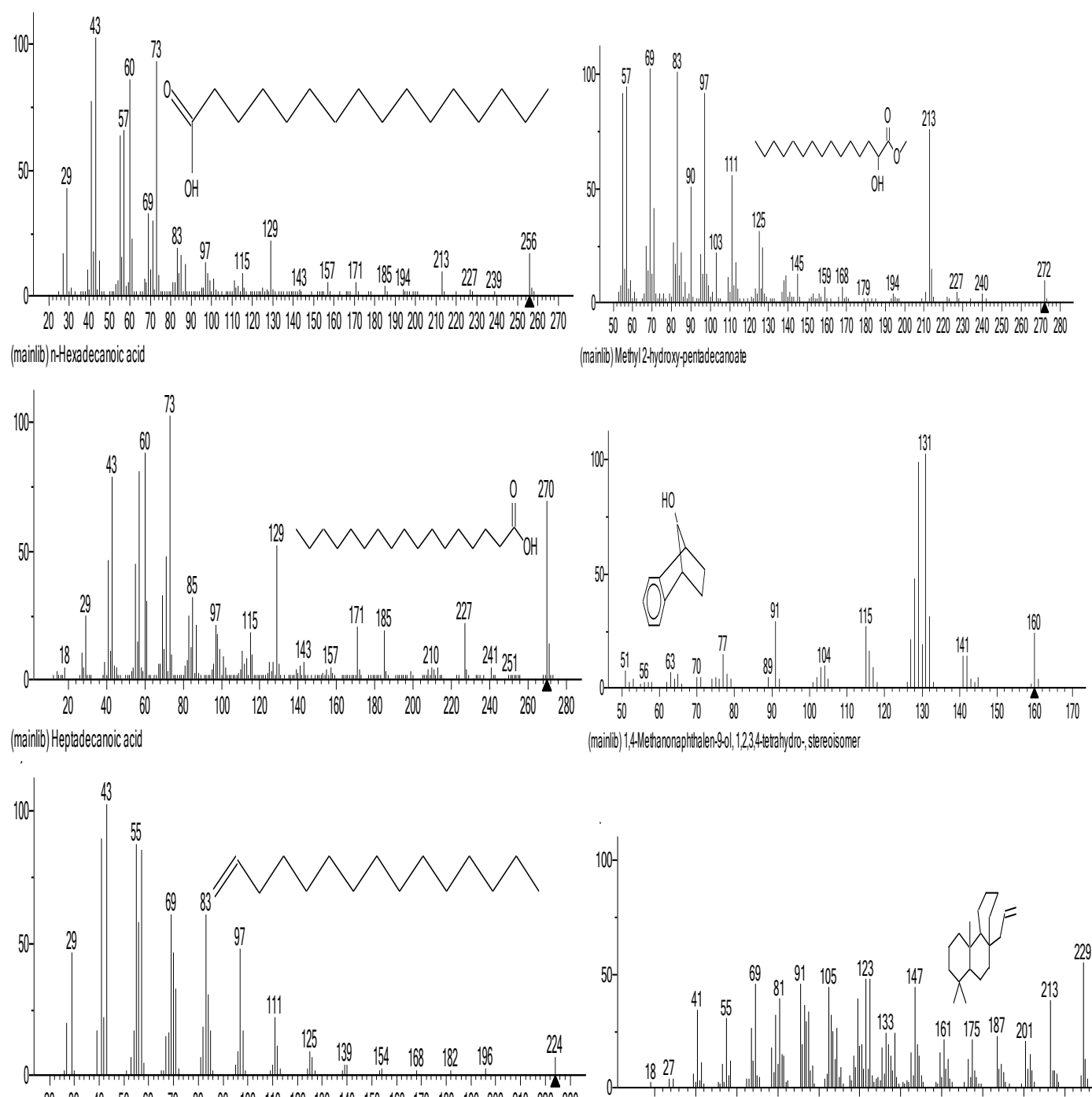

$2030 \quad 4050 \quad 6070 \quad 8090100110120130140150160170180190200210220230$ (mainili) Cetene
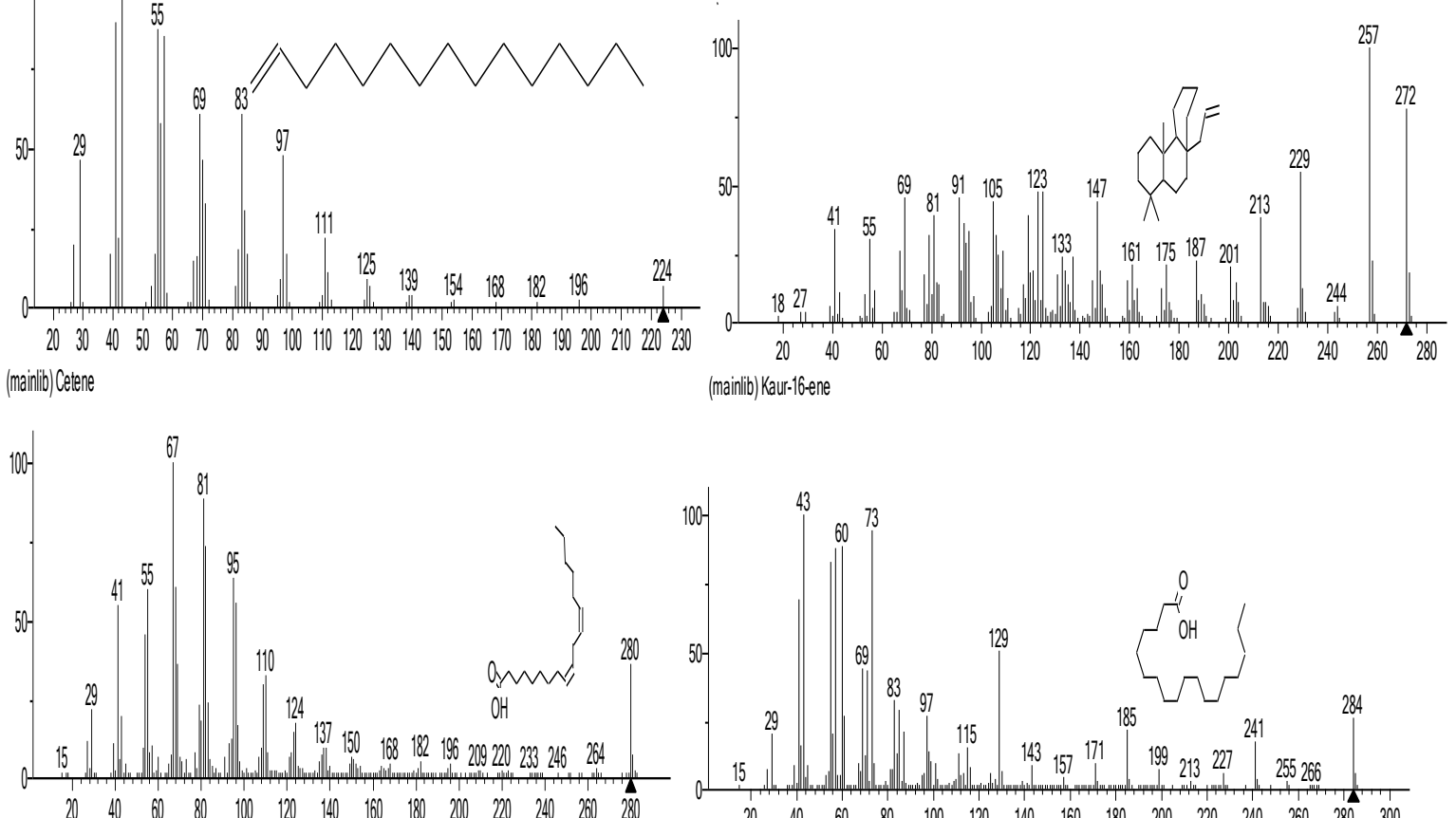

(mainili) 9,12-0ctadecadienocracid (2,2).
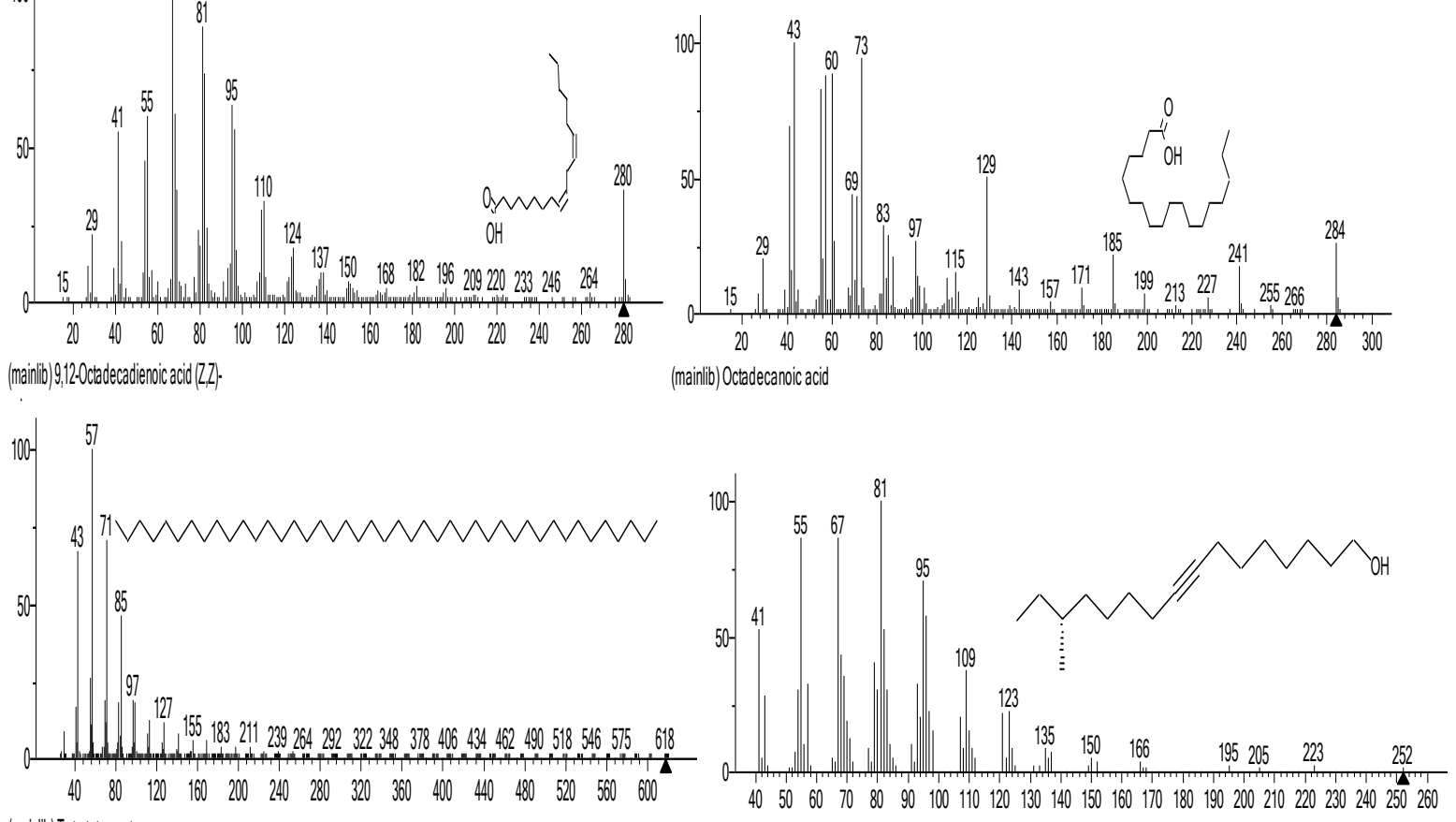
(manilib) Tetratetracontane (mainilib)(R)-(-1-14-Metyy-8--hexadecyn-1-0l 

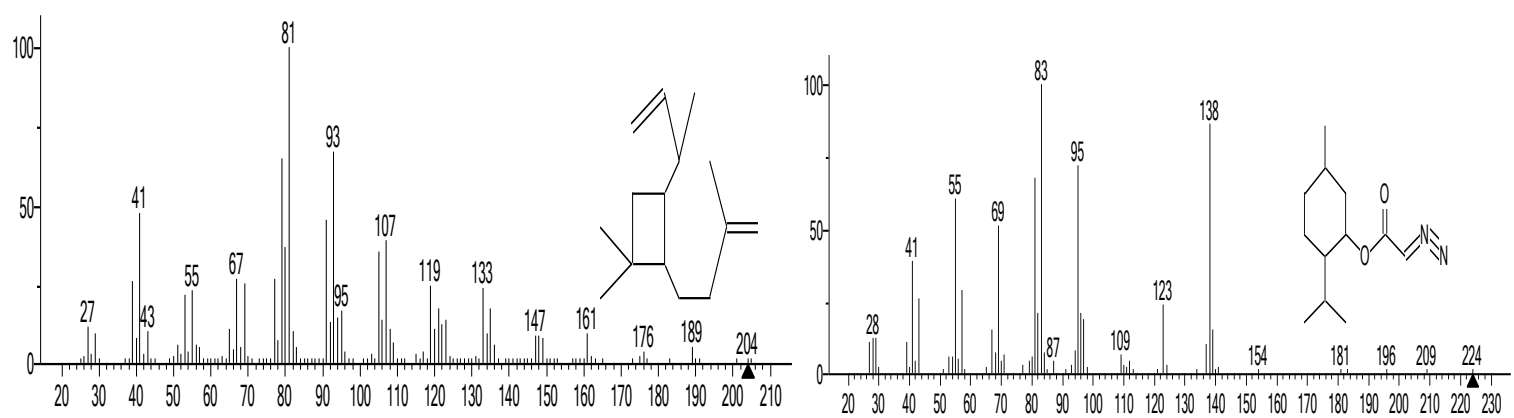

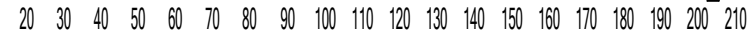
(mainilib) Bicyclo[5.20]nonane, 4-4methylene-28,8-4timethy-2-viny-. (mainili) Diazoacetic acid, 2-isopopoyy.5-methylcyclohexy) ester
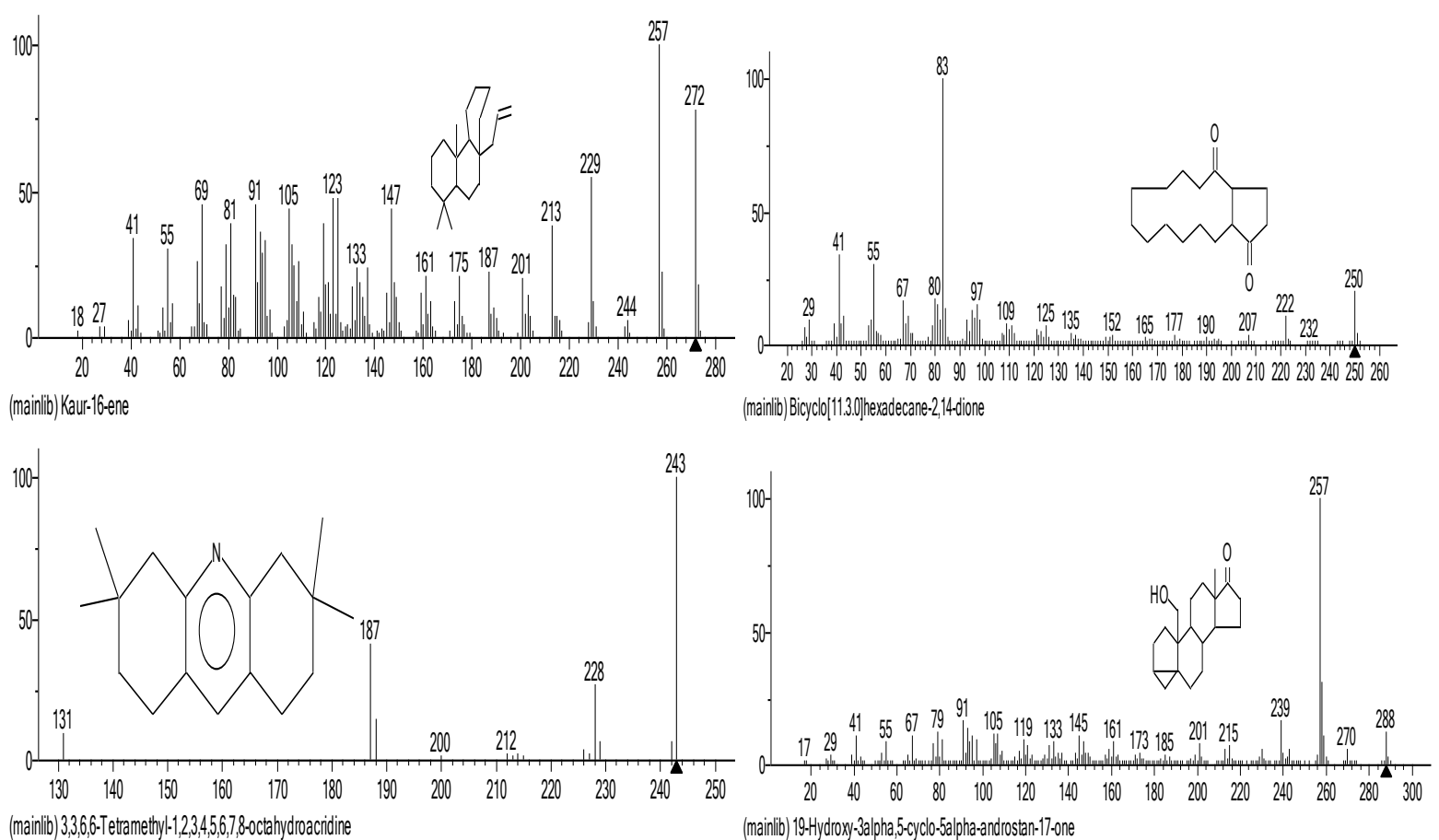

(mainlib) 3,3,6,6-Tetamethy-1,2,3,4,5,6,7,8-0ctahydroacridine
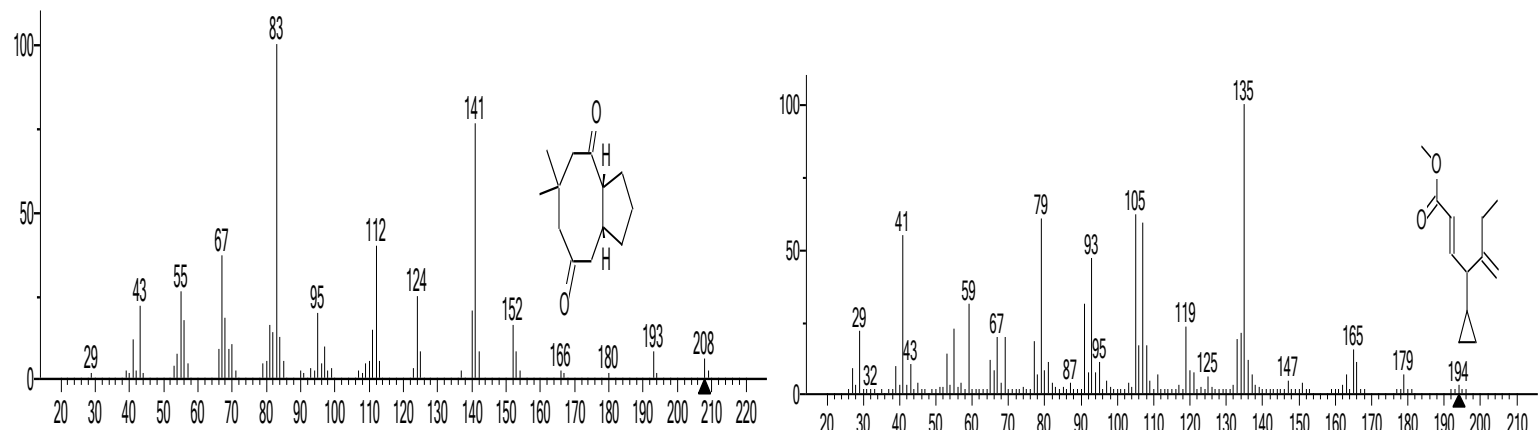

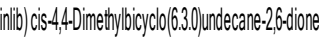

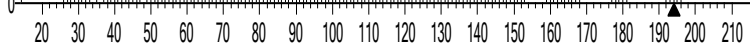
(mainiti) 2-Hepetenoic acid,4-4cyclopropy-5-5methylene; methyl esier, (E).

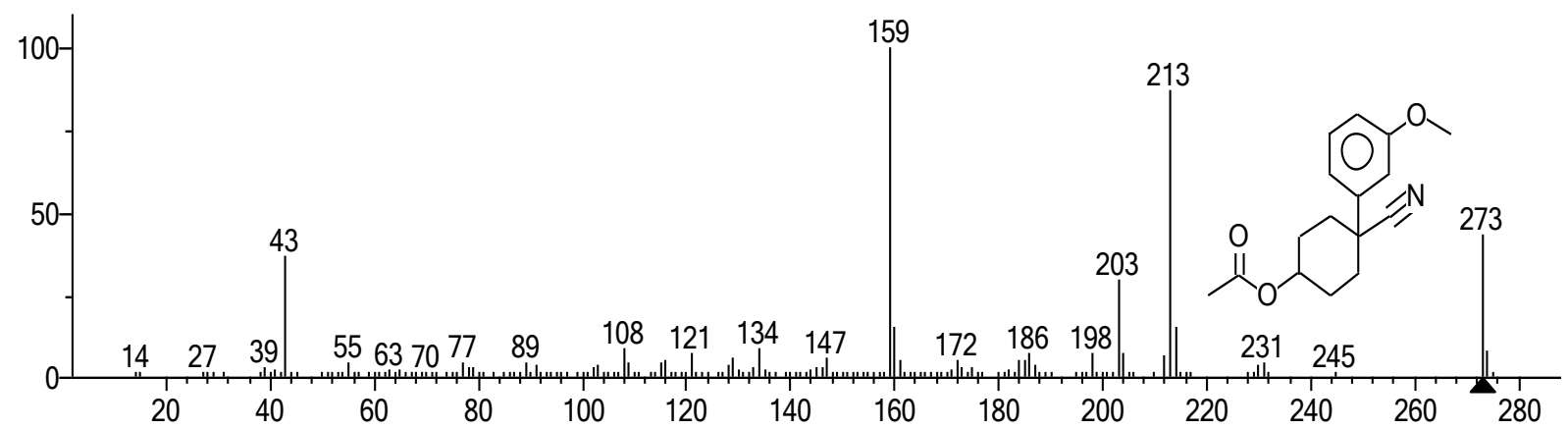

(mainlib) cis-4-Acetoxy-trans-1-(m-methoxyphenyl)cyclohexanecarbonitrile

Figure2. GC-MS of Mass Spectra Ageratum conyzoides whole plant chloroform extract 


\section{COnClusion}

This present study revealed the presence of seventy one components in Ageratum conyzoides through GC-MS analysis. The plant specie used in this study has been discovered to possess promising medicinal potentials. This study has suggests that chloroform extract contain more of the phytochemicals. The presence of bioactive components justifies the use of the plant for various ailments by traditional practioners. In view of the medicinal importance associated with the phytocompounds found in this plant, further investigation should be carried out in order to purify, characterize the structure of these bioactive compounds and enhance their potentials as drugs.

\section{REFERENCES}

[1] Macia, M.J., Garcia, E. and Vidaure, P.J. (2005). An ethno botanical survey of medicinal plants commercialized in the markets of La Paz and El Alto, Bolivia. Journal of Ethnopharmalogy; 97(2): 337-35.

[2] Kong, C.H. (2006). Allelochemicals from Ageratum conyzoides L. and Oryza Satiiva L. and their effects on related pathogens. Allelopathy J.; 14: 5-13.

[3] Okunade, A.L. (2002). Review: Ageratum conyzoides L (Asteracease)”. Fitoterapia; 73: 1-16.

[4] Igoli, J.O., Ogaji, O.G., Tor-Anyin, T.A. and Igoli, N.P. (2005). Traditional medicine practice among the Igede people of Nigeria (part II). Afri. J. Trad. Comp. Alt. Med.; 2:134-152.

[5] Oladejo, O.W., Imosemi, I.O., Osuagwu, F.C., Oluwadara, O.O., Aiku, A., Adewoyin, O., Ekpo, O.E., Oyedele, O.O. and Akang, E.E.U. (2003). Enhancement of Cutaneous Wound Healing by Methanoic Extracts of Argeratum conyzoides in the Wister Rat. African Journal of Biomedical Research; 6(1): 27-31.

[6] Aja, P.M., Enechi, O.C., Ozougwu, V.E., Onya-Mmaghiri, E.A., Agu, K.A., Ali-Ikechukwu, A. and Nweke, O.L. (2016). Phytochemical Composition, Gas Chromatography-Mass Spectrometric (GC-MS) Analysis and Anti-Bacterial Activity of Ethanol Leaf - Extract of Ageratum conyzoides. African Journal of Basic \& Applied Science; 8(1): 34-40.

[7] Raheala, J., Muhammad, S., Amer, J. and Muhammad, A. (2008). Microscopic Evaluation of the Antimicrobial Activity of Seed Extracts of Moringa oleifera. Pakistan Journal of Botany; 40(4): 1349-1358.

[8] Wandji, J., Bissangou, M.F., Ouambra, J.M., Silou, T., Abena, A. and Keita, A. (1996). Invasive Alien Plants: An Ecological Appraisal for the Indian Subcontinent. Fitoterapia; 67: 427.

[9] Ekundaya, O., Laasko, I. and Hiltunen, R. (1988). Composition of Ginger (Zingiber officinale Roscoe) Volatile OilS from Nigeria. Flav. Fragr J.; 3: 85-90.

[10] Lv, S.D.; WU, Y.S.; Li, G.W..; Xu, Y.Q; Liu, L.; and Meng, Q.X. (2014). Comparative Analysis of pu-erh Tea and Fuzliuan Teas by fully Automatic Headspace solid-phase micro extraction coupled with Gas Chromatography-Mass Spectrometry and Chemometric Methods. Journal of Agricultural and Food Chemistry; 62: 1810-1818.

[11] Boutekedirect, C., Bentahar, F., Elabbes, R. and Bessiere, J.M. (2003). Extraction of Rosemary Essential oil by Steam Distillation and Hydrodistillation. Flavour and Fragrance Journal; 18: 481-484.

[12] Bourgou, S., Tammar, S., Nidhal, S., Khawla, M., and Kamel, M. (2015). Phenolic Composition, Essential oil, and Antioxidant Activity in the Aerial Part of Artemisia Herba-Alba from Several Provenances: A Comparative Study. International Journal of Food Properties; 19 (3): 549-563.

[13] Igwe, O.U. and Okwu, D.E. (2013). GC-MS Evaluation of Bioactive Compounds and Antibacterial Activity of the Oil Fraction of the Stem Bark of Brachystegia eurycoma.Int. J. Chem Sc.; 11(1): 357-371.

Citation: A. Ahuchaogu et al., "GC-MS Analysis of Bioactive Compounds from Whole Plant Chloroform Extract of Ageratum conyzoides", International Journal of Medicinal Plants and Natural Products (IJMPNP), vol. 4, no. 2, pp. 13-24, 2018. http://dx.doi.org/10.20431/2454-7999.0402003

Copyright: () 2018 Authors. This is an open-access article distributed under the terms of the Creative Commons Attribution License, which permits unrestricted use, distribution, and reproduction in any medium, provided the original author and source are credited. 